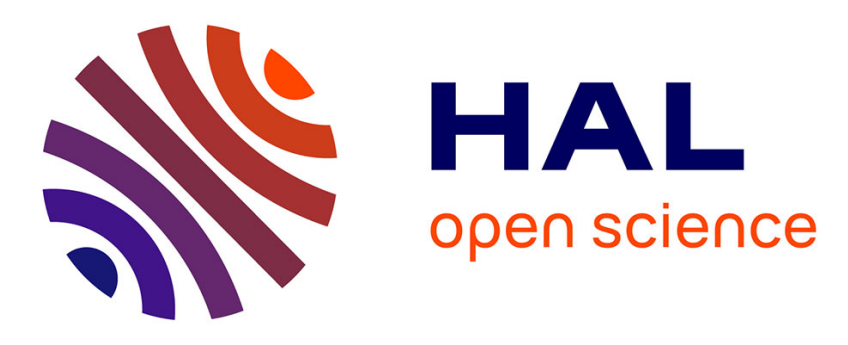

\title{
Energy-filtered electron microscopy for imaging core-shell nanostructures
}

Cyril Langlois, T. Oikawa, Pascale Bayle-Guillemaud, Christian Ricolleau

\section{To cite this version:}

Cyril Langlois, T. Oikawa, Pascale Bayle-Guillemaud, Christian Ricolleau. Energy-filtered electron microscopy for imaging core-shell nanostructures. Journal of Nanoparticle Research, 2008, 10 (6), pp.997-1007. 10.1007/s11051-007-9329-0 . hal-02161263

\section{HAL Id: hal-02161263 \\ https://hal.science/hal-02161263}

Submitted on 20 Jun 2019

HAL is a multi-disciplinary open access archive for the deposit and dissemination of scientific research documents, whether they are published or not. The documents may come from teaching and research institutions in France or abroad, or from public or private research centers.
L'archive ouverte pluridisciplinaire HAL, est destinée au dépôt et à la diffusion de documents scientifiques de niveau recherche, publiés ou non, émanant des établissements d'enseignement et de recherche français ou étrangers, des laboratoires publics ou privés. 


\title{
Energy filtered electron microscopy for imaging core-shell
}

\section{nanostructures}

\author{
Cyril T. Langlois ${ }^{* t}$, Tetsuo Oikawa ${ }^{\circ}$, Pascale Bayle-Guillemaud ${ }^{\S}$ and Christian Ricolleau ${ }^{\dagger t}$ \\ ${ }^{\dagger}$ Laboratory for Materials and Quantum Phenomena, University Paris 7, 4 rue Elsa Morante, 75205 Paris, FRANCE. \\ ${ }^{*}$ Laboratory for Solid State Physics, ESPCI, 10 rue Vauquelin 75005 Paris, FRANCE. \\ JEOL Ltd, 1-2 Musashino 3-Chome, Akishima, Tokyo, 196-8558 JAPAN. \\ ${ }^{\S}$ DRFMC-CEA, 38000 Grenoble FRANCE. \\ *Corresponding author. \\ E-mail: cyril.langlois@paris7.jussieu.fr. Tel: +33157276996 Fax: +33157276241.
}

\begin{abstract}
CuAg}$ core-shell nanoparticles are synthesized by ultra-high vacuum thermal evaporation. We show on this system how the Energy-Filtered Transmission Electron Microscopy (EFTEM) technique allows one to improve the characterization by precisely pointing out the formation of core-shell arrangements in bimetallic nanoparticle assemblies. A criterion to measure the shell thickness from EFTEM images on unique core-shell nanoparticles is defined, that can be used for core-shell nanoparticles of any sizes, with shell thicknesses over $1 \mathrm{~nm}$. It is based on the intensity variation along a line drawn across a core-shell nanoparticle on a EFTEM image. This criterion has been validated by a close comparison of the shell thickness measurements performed in this work and the ones obtained by acoustic micro-Raman spectroscopy. Using this criterion, we report a strong correlation between the size of the $\mathrm{Cu}$ cores and the formation of the core-shell arrangements in the nanoparticle assembly studied in this work. The influence of the Cu core shape is also evidenced. The characterisation of such systems using High Resolution TEM (HRTEM) is also discussed.
\end{abstract}

Keywords: Energy filtered transmission electron microscopy ; nanoparticles ; core-shell system ; crystal growth. 


\section{Introduction}

Core-shell nanostructures have recently attracted much attention, from both experimental and theoretical point of views, since it is possible to enhance or to design new physical and chemical properties that cannot be obtained in the single components nanoparticles. It is also possible, by modifying the thickness of the shell to tailor these properties in a certain range. Coreshell nanoparticles have been used for catalysis applications, optical and magnetic properties. For the catalysis applications, the association of two different metals offers higher catalytic activity, better selectivity of catalytic reactions and stability (Sao-Joao et al., 2005; Skarman et al., 2002; Toshima, 2000; Toshima et al., 1992). In the case of magnetic properties, core-shell nanostructures have been developed to obtain nanoparticles with individual improved magnetic properties by reducing the direct interaction between the nanoparticles (Favre et al., 2004; Zeng et al., 2004). In the optical domain, semiconductor core-shell nanostructures have been synthesized to enhance photoluminescence by the passivation of the core material with a shell having a higher band gap energy (Chen et al., 2003; Reiss et al., 2002), to improve stability against photochemical oxidation and to perform band structure engineering (Correa-Duarte et al., 1998; Kim et al., 2003). Metal/oxide and metallic core-shell nanostructures have also been developed in order to modify the surface plasmon resonance energy to a given value by varying the relative composition of the core and the shell as well as their relative size (Basu \& Chakravorty, 2006; Gaudry et al., 2003; Zhu et al., 2004). Finally, some recent papers report the use of core-shell nanocomposite materials formed with metal, oxide and/or semiconductor in order to combine magnetic and optical properties in a single nanostructure (Kim et al., 2005; Lu et al., 2002).

In all such applications, the knowledge of the morphology as well as the precise thickness of the shell are key parameters in order to understand the physico-chemical properties of the nanostructures. Several techniques have been used to study these nano-objects. These techniques can be classified in two categories: the ones that give access to direct imaging of the core-shell 
nanostructures and the spectroscopic techniques which give indirect information on the nanostructures but in a more statistical way. Transmission Electron Microscopy (TEM) is one of the best tools to image such nanostructures. Among the various TEM techniques, the most common ones to study single objects are the High Resolution Transmission Electron Microscopy (HRTEM) and the Scanning Transmission Electron Microscopy (STEM) imaging techniques. While the first one has several limitations (low sensitivity to chemistry, zone axis orientation, etc.) for the characterization of core/shell nanostructures the second one has given very good results in the AuPt and AuAg systems (Garcia-Gutierrez et al., 2004; Li et al., 2005).

In the domain of spectroscopy, Raman (Hodak et al., 2000; Sader et al., 2002), EXAFS (Extended X-ray Absorption Fine Structure) (Harada et al., 1992) and optical spectroscopies (Lu, et al., 2002; Huang et al., 2004; Yang et al., 2005) have been widely used. Since the interpretations of the data are model dependent, they have to be used in close connection with, at least, one of the imaging techniques cited above (Cazayous et al., 2006).

In this paper, we have studied by Energy Filtered TEM (EFTEM) the CuAg core-shell nanostructure. A core-shell growth is expected according to thermodynamical arguments: silver and copper being immiscible elements $(0.1 \%$ solid solution at $298 \mathrm{~K})$ and a lower surface energy for $\mathrm{Ag}\left(1210 \mathrm{~mJ} / \mathrm{m}^{2}\right.$ for Ag compared to $2130 \mathrm{~mJ} / \mathrm{m}^{2}$ for $\left.\mathrm{Cu}\right)$ favor the surface silver segregation. These values are averaged from $\{111\},\{110\}$ and $\{100\}$ surfaces energies (Vitos et al., 1998). The chemical mapping by EFTEM has been widely used for the characterisation of multilayered materials. This is a perfect tool to study core-shell nanostructures since it produces a chemical map of the specimen on sufficiently large area that allows to obtain statistical data on the specimen. To our knowledge, the EFTEM technique is actually not enough popular in the materials science community and needs further attention because of its high performance to image complex nanoobjects at a nanometer resolution. These experiments have been coupled to HRTEM observations in order to study the structural properties of the core-shell configurations at the atomic scale. 


\section{Experimental methods}

\section{CuAg core-shell synthesis}

Core-shell $\mathrm{CuAg}$ nanostructures are fabricated by successive deposition of $\mathrm{Cu}$ and $\mathrm{Ag}$ under ultra-high vacuum conditions directly onto carbon coated grids. The deposition method is thermal evaporation from two crucibles containing respectively $\mathrm{Cu}$ and $\mathrm{Ag}$. The substrate is an ultra-thin amorphous carbon film (thickness about $4 \mathrm{~nm}$ ) transparent to electron beam and supported by a holey carbon microscopy grid. This kind of substrate was chosen because of the importance of substrate thickness in the results presented hereafter and also because it can be used by almost everyone involved in nanoparticles fabrication, including both chemists and physicists.

During the deposition of $\mathrm{Cu}$ and $\mathrm{Ag}$, the substrate is heated at a temperature of $400^{\circ} \mathrm{C}$. After this operation, the nanoparticles are annealed during half an hour at the same temperature in ultrahigh vacuum. Pressure inside the deposition chamber is below 10-7 Torr. Typically, evaporation rates for $\mathrm{Cu}$ and $\mathrm{Ag}$ were around $0.25 \mathrm{~nm}$ per minute in nominal thickness. Since the wetting of metallic species on amorphous carbon is low (Campbell, 1997), nanoparticles are formed instead of a film on the substrate and the nominal thickness indication does not obviously correspond to the size of the particles. For $\mathrm{CuAg}$ nanoparticles, two different core-shell nanoparticles were synthesized: (i) sample A obtained by a deposition of $6 \mathrm{~nm}$ of $\mathrm{Cu}$ followed by a deposition of $3 \mathrm{~nm}$ of $\mathrm{Ag}$, and (ii) sample B obtained by a deposition of $2 \mathrm{~nm}$ of $\mathrm{Cu}$ followed by a deposition of $1 \mathrm{~nm}$ of Ag.

\section{Energy filtered TEM technique and application to chemical mapping}

The basic principle of the Electron Energy Loss Spectroscopy (EELS) consists in the energy dispersion of the electron beam with a magnetic prism after the specimen in order to obtain a spectrum of the electron energy losses (Egerton, 1996). Energy filtered imaging (EFTEM) can be obtained by introducing a slit in the energy dispersive plane along the optical path and hence 
selecting only electrons having lost a specific part of their energy by inelastic interactions with the different elements of the specimen. By optically forming images with the energy filtered electrons, it is then possible to obtain chemical maps of the region of interest (Reimer, 1995; Shindo \& Oikawa, 2002).

For core-shell nanoparticles, lateral (projected) dimensions of the Ag shell are small, and the thickness is also limited along the optical axis. These limitations result usually in poor signal-tonoise ratio (SNR) for the images and may be the restrictive criterion for applying the EFTEM technique to nanostructured core-shell particles. This is the reason why we used an ultra-thin carbon film as a substrate for the nanoparticles deposition, in order to minimize its contribution to the filtered image. Choosing an commercial amorphous carbon substrate is interesting for characterizing nanoparticles synthesized by chemical routes: several procedures exist to transfer the nanoparticles from the solvent to the surface of the thin carbon film without too much aggregation (Zeng, et al., 2004; Yang, et al., 2005).

EELS spectra have been first acquired on the B sample, with the thinnest Ag layer, to get insight for the EFTEM parameters, that is, at which edges the selecting slit should be exactly placed for constructing the chemical maps for $\mathrm{Ag}$ and $\mathrm{Cu}$, as well as the appropriate energy width of the slit. A large area including many particles has been selected by decondensing the electron beam. Experimental $\mathrm{Cu}$ and $\mathrm{Ag}$ electron energy-loss spectra are shown on Figure 1a and $1 \mathrm{~b}$ respectively. The shape of the $\mathrm{Cu}$ edge corresponds to the reference given by Ahn and Krivanek (Ahn \& Krivanek, 1983), with a smooth edge located at $920 \mathrm{eV}$ ( $\mathrm{L}_{2,3}$ edges), followed by a second part slowly decreasing. The $\mathrm{L}_{1}$ core-loss absorption edge is clearly visible at $1100 \mathrm{eV}$. The shape of the spectrum is typical from non-oxidized copper (Shindo \& Oikawa, 2002). Due to the small quantity of silver with respect to copper (1:2) together with the formation of shells instead of clusters, the Ag spectrum is far more diluted into the background. 
The microscope used for this study was a JEM $2100 \mathrm{~F}$ working at $200 \mathrm{keV}$, equipped with a high-resolution pole piece (point-to-point resolution of $0.19 \mathrm{~nm}$ ) and a post-column GIF 2001 (Gatan Imaging Filter). Chemical maps have been acquired by the three-window technique (Reimer, 1995) using the GATAN Digital Micrograph software. The optimum conditions are realized using a slit width of $30 \mathrm{eV}$ with the three windows centred at 340, 380 and $420 \mathrm{eV}$ for $\mathrm{Ag}$, and 850, 900 and $950 \mathrm{eV}$ for $\mathrm{Cu}$.

\section{Chemical and structural study}

\section{Sample A - Chemical mapping}

Conventional TEM images in bright field mode are commonly used to determine statistically the shape and the size of the nanoparticles. However, this type of images gives no insight on the chemical nature of the nanoparticles. In this work, chemical maps of $\mathrm{Ag}$ and $\mathrm{Cu}$ are obtained by performing EFTEM experiments on nanoparticles formed on sample A. Figure 2 shows a set of three EFTEM images revealing the core-shell structure of the considered area. On the Ag map (Figure 2a) and the $\mathrm{Cu}$ map (Figure 2b), white pixels correspond to $\mathrm{Ag}$ and $\mathrm{Cu}$ signals, respectively, on the same area. The complementarity between the $\mathrm{Cu}$ and $\mathrm{Ag}$ localization is well convincing. Figure 2c shows a color image compiling $\mathrm{Ag}$ and $\mathrm{Cu}$ maps together, which gives a strong evidence of the formation of core-shell arrangements on sample A.

\section{Criterion for shell thickness measurements}

The criterion that will be used to measure the Ag shell thickness on the EFTEM images has to be clearly defined before starting a statistical study of the EFTEM images. To address this point, we consider the intensity profiles drawn on an Ag EFTEM image of sample A across (i) a core-shell nanoparticle (Figure 3a, left) and (ii) a pure Ag nanoparticle (Figure 3a, right). First of all, we can notice that the Ag signal on the core-shell intensity profile is lower near the centre of the nanoparticle than on the edges, where the profile exhibits two intense peaks with different 
decreasing behavior depending on whether the inner or the outer side of the nanoparticle. These two observations can be explained using a simple geometrical model of the nanoparticle shape: the cores are assumed to be half ellipsoids, truncated by the substrate, and silver consists in an homogeneously thin layer covering the core. The half ellipsoidal morphology is considered here because of (i) the weak interactions between copper and the amorphous substrate, (ii) high diffusion of metallic species on the heated substrate and (iii) coalescence events during the growth, giving rise to elliptic morphologies in the plane of the substrate. Figure $3 b$ shows a cross section of such a modelled core-shell nanoparticles (black lines). The Ag thickness crossed by the electron beam (which is considered perpendicular to the substrate) as a function of the position is also represented on the figure (grey line). A larger Ag thickness crossed by the electron beam will results in a larger number of inelastic interaction events. This is the reason why the intensity profile will be higher on the edges of the core and smaller in the middle of the nanoparticle. As a consequence, the two peaks match the maximum projected Ag thickness visible on the model. The decreasing behaviors on both sides of the peaks also correspond to the variation of the Ag thickness crossed by the electron beam. It is important to note that the global shape of the intensity profile (double-peaked and non-zero intensity at the centre) is the same if we consider a spherical shape, an ellipsoidal shape with any semi-axes values, with any height of truncature by the substrate.

A question remains: where stops the Ag layer on the edges of the core-shell nanoparticle? It seems reasonable to consider that when the intensity becomes comparable to the noise level of the image, the limit of the nanoparticle is reached. The error on the measurement then depends on the shape of the silver layer and the SNR of the images.

From these considerations, the criterion chosen to measure with a good approximation the Ag shell thickness was defined as the lateral extension between the maximum intensity of the peak and the limit of the area for which the intensity becomes comparable to the standard deviation of the noise level of the image. An example is shown on the core-shell intensity profile of Figure 3a left (black area). 
Profiling also allows differentiating between core-shell nanoparticles and pure Ag nanoparticles on EFTEM images. On the EFTEM image of Figure 3a, a second distribution of very small Ag nanoparticles appears between the large $\mathrm{CuAg}$ clusters. For these pure Ag nanoparticles, the contrast variation across the particle (Figure 3a, right) is obviously different from the core-shell profile of Figure 3a, left. The results of the shell thickness measurements using the criterion described here have been confirmed by a more global technique. In a previous work (Cazayous, et al., 2006), we measured by micro-Raman spectroscopy the shell thicknesses in an assembly of $\mathrm{CuAg}$ core-shell nanoparticles precisely located on a labelled microscopy grid. The measurements were obtained using the Lamb theory to extract the average Ag layer thickness from acoustic breathing resonance of the core-shell nanoparticles, with an accuracy of $0.3 \mathrm{~nm}$. The results obtained on the very same particle population using a global and a local technique were identical and this gives us a strong confidence in the EFTEM measurement criterion defined in this work.

\section{Core size and shell thickness measurements}

The size distribution on CuAg sample A, presented on Figure 4a, exhibits a bimodal size distribution centred on $4.5 \mathrm{~nm}$ (FWHM $3.9 \mathrm{~nm}$ ) for the first peak and $20.5 \mathrm{~nm}$ (FWHM $11.6 \mathrm{~nm}$ ) for the second. This corresponds to the core-shell $\mathrm{CuAg}$ population combined with the small pure Ag nanoparticles. Such a polydisperse distribution is common when thermal evaporation is used to synthesize the nanoparticles. The shell thickness distribution (Figure 4b) obtained from EFTEM images is centred on $5 \mathrm{~nm}$ (FWHM $2.2 \mathrm{~nm}$ ), with values ranging between $3 \mathrm{~nm}$ and $7 \mathrm{~nm}$.

As it can be seen on Figure 2c, different configurations are formed on the substrate. Perfect $\mathrm{CuAg}$ core-shell nanoparticles are visible, together with partially segregated ones and pure Ag nanoparticles. It is interesting to note that segregation occurs for the largest nanoparticles, contrary to the smallest ones which are in a perfect core-shell arrangement. This point will be developed later in this paper. 


\section{Moiré effect}

The complete capping of the $\mathrm{Cu}$ core by the $\mathrm{Ag}$ shell is evidenced by a well-known optical effect called 'moirés' (Figure 5). Large dark stripes appear on bright field TEM images when interferences occur between two slightly different spatial frequencies (in orientation and/or spacing) in the reciprocal space. Here, the two sets of fringes are the (111) fringes from $\mathrm{Cu}$ and $\mathrm{Ag}$ and they are oriented in the same direction because of the epitaxial relationship between the two crystals. This epitaxial relationship can be seen on the power spectrum on the inset of Figure 5, on which Ag and $\mathrm{Cu}$ crystals are oriented along the same [110] zone axis of the Face Centred Cubic (FCC) structure, with the same radial orientation of the four equivalent 111 reflections of the two crystals. A set of moiré fringes is then formed, normal to $\Delta \boldsymbol{g}=\boldsymbol{g}_{111}^{C_{u}}-\boldsymbol{g}_{111}^{A_{g}}$, with spacing equal to:

$$
d_{\text {Moiré }}=\frac{1}{\Delta \boldsymbol{g}}=\frac{d_{111}^{A g} d_{111}^{C u}}{d_{111}^{A g}-d_{111}^{C u}}
$$

By taking $d_{111}^{c u}=0.2087 \mathrm{~nm}$, the moiré pattern periodicity $\left(\mathrm{d}_{\text {moiré }}=1.7+/-0.15 \mathrm{~nm}\right)$ gives a value of $0.237 \mathrm{~nm}$ for $d_{111}^{A g}$ in the epitaxial layer, which gives an Ag lattice parameter equals to $a=0.410 \pm 0.06 \mathrm{~nm}$, showing that the silver layer has recovered the bulk Ag lattice parameter $\left(a_{\text {bulk }}=0.4086 \mathrm{~nm}\right)$. This means that the thickness of the Ag layer is greater than the critical thickness below which the accommodation of the core-shell lattice misfit by elastic strain would occur.

\section{Sample B - Chemical mapping and measurements}

From Figure 2c, it is worth noting that the core-shell arrangement is found for the smallest nanoparticles $(<12 \mathrm{~nm})$ of the distribution. Over this limit, most of the CuAg nanoparticles do not present the core-shell configuration. The size of the pre-existing $\mathrm{Cu}$ cores being unambiguously an important parameter in the formation of the core-shell arrangement, we turned to the synthesis of a core-shell system with smaller cores. Controlling the size of the $\mathrm{Cu}$ nanoparticles can be done by 
two different means. The first way would be to lower the substrate temperature during the formation of the $\mathrm{Cu}$ core, decreasing the $\mathrm{Cu}$ diffusion on the surface. $\mathrm{Cu}$ atoms would be then fixed more efficiently on the nucleating sites, resulting in an increased number of particles per unit area, and subsequently smaller particle sizes. The other mean is to decrease the quantity of matter during deposition, keeping constant all the other parameters, especially the substrate temperature. The density of particles per square nanometer remains the same, and the mean nanoparticles size decreases because of the lack of atoms available for the growth.

For the sample B, the same ratio 2:1 between the two elements has been kept but with three times less copper and silver deposited (namely $2 \mathrm{~nm}$ of $\mathrm{Cu}$ and $1 \mathrm{~nm}$ of $\mathrm{Ag}$ in nominal thickness). These growth conditions have resulted in smaller core-shell nanoparticles with a smaller shell thickness.

The same procedure as for the sample A has been used on sample B to obtain the size distribution of the $\mathrm{CuAg}$ and $\mathrm{Ag}$ nanoparticles as well as the shell thicknesses (Figure 6). First, the observation made on sample A is confirmed: one can note that perfect core-shell nanostructures are more frequently formed on the substrate, compared to sample A (Figure 6a). The size distribution on CuAg sample B presents a bimodal size distribution centred on $5 \mathrm{~nm}$ (FWHM $9.5 \mathrm{~nm}$ ) for the first peak and $17 \mathrm{~nm}$ (FWHM $5.0 \mathrm{~nm}$ ) for the second. The shell thickness distribution obtained from EFTEM images is centred on $2 \mathrm{~nm}$ (FWHM $0.5 \mathrm{~nm}$ ), with values ranging between $1 \mathrm{~nm}$ and 3.5 nm. This could be explained by a smaller initial $\mathrm{Cu}$ core size, suggesting a critical core size over which silver segregation is promoted. The origin of a critical core size may be linked to the way silver grows on the $\mathrm{Cu}$ nanoparticles. From crystal growth theory, it is well known that the growth rate is different depending on the Miller indices of the face exposed to incoming species. On the other hand, $\mathrm{Cu}$ nanoparticles exhibits three different morphologies depending on their size: icosahedral below $4.5 \mathrm{~nm}$, decahedral between 4.5 and $7 \mathrm{~nm}$ (Mottet et al., 2004) and FCC Wulff shape above $7 \mathrm{~nm}$ (Henry, 1998). Icosahedral and decahedral shapes present principally (111) faces. As a consequence, the growth of the Ag shell will tend to be isotropic, and hence the thickness will 
be homogeneous all around the $\mathrm{Cu}$ cores. Further experiments are currently under progress to detail this result.

\section{HRTEM core-shell arrangement characterization}

In addition to the EFTEM technique, it is interesting to consider the use of HRTEM to characterize the core-shell configurations on the substrate. Figure 7a shows a HRTEM image acquired on a core-shell nanoparticle. This image has been Bragg-filtered to compute a new image on which the contrast corresponds to the localization of $\{200\}$ Ag lattice fringes (Figure $7 \mathrm{~b}$ ). The radius of the mask for the filter has been chosen to ensure a resolution of one lattice fringe (i.e. 0.2 $\mathrm{nm})$. The same core-shell nanoparticle has been observed by EFTEM, and the corresponding image is presented on Figure 7c. By comparing the two images (Figure $7 \mathrm{~b}$ and $\mathrm{c}$ ), it is clear that trying to localize silver from the Bragg filtered image is totally misleading if we refer to the EFTEM image.

The HRTEM images can give wrong chemical information because of both technical artefacts and limitations due to the sample. From a technical point of view, the best contrast conditions are usually obtained by defocusing the objective lens of the microscope in order to adjust the transfer function of the microscope to the so-called Scherzer conditions. Fringes delocalization occurs in this case. Moreover, $\mathrm{Ag}$ and $\mathrm{Cu}$ superimposition effects influence dramatically the image contrast. To extract quantitative information from HRTEM images in such case, one has to carry out image contrast simulations by continuously varying the thicknesses of the two materials, together with the focus of the objective lens (through-focus series). Conditions for which the Ag lattice fringes vanish completely can be identified by this way. Concerning sample limitations, the nanoparticles may not be perfectly in zone axis orientation due to the amorphous substrate, which disturbs the formation of lattice interference fringes for both $\mathrm{Ag}$ and $\mathrm{Cu}$. It is also assumed that the Ag layer is perfectly relaxed to its own bulk lattice parameter, without any strain effects from the underlying $\mathrm{Cu}$ lattice. Finally, if the Ag shell is locally slightly disoriented, the intensity will fall down in the Bragg-filtered image, and will result in misleading chemical information. To 
summarize, it is worth noting that obtaining chemically interpretable HRTEM images is rather difficult, particularly if statistical analysis is required. In addition, for core-shell systems, in which the lattice parameters are very close, high resolution lattice contrasts appear the same on the images, and this technique is useless to distinguish between the core and the shell.

This pessimistic presentation is tempered by the fact that, among the billion particles accessible to observation on the microscopy grid, a significant number will present good imaging conditions without most of the limitations exposed above. Hence, a good result is a question of time of experiment, but in no case HRTEM can replace EFTEM statistical efficiency for determining the different chemical arrangements.

\section{Discussion}

For the core-shell systems, characterizing statistically the segregation of silver by means of HRTEM only would have been a giant task. On the contrary, EFTEM imaging provides an answer on silver localization for each particle in the field of view of the recording camera, in an acquisition time of few tens of seconds. According to the arguments developed in the previous parts, the preeminence of EFTEM over HRTEM is clearly demonstrated in case of chemical core-shell nanoparticles characterisation.

For a large part of research teams working on core-shell nanostructures, either synthesized by chemical or physical route, the EFTEM technique could consist in a highly valuable help for characterizing such nano-objects. Another argument for the EFTEM promotion is the large number of filters available through the world, easily accessible through collaborations.

More generally, even if intense research efforts have been conducted on global optical method to infer the structural nature (core-shell or not) of an assembly of nanoparticles (Chen, et al., 2003; Lu, et al., 2002), a local characterisation using EFTEM or other TEM techniques must 
never be ruled out in order to take into account the potential complexity of layered nanoparticles as well as possible segregation of one species into a second population of pure element clusters, together with inhomogeneities within the specimen (Cazayous et al., 2006).

The successful use of the EFTEM technique to characterize a given system is also dependent on some experimental considerations. The pertinence of the elemental mapping is based on a correct background subtraction to remove from the image the contribution of electrons coming from interactions with other elements and substrate. This correction may be difficult to achieve when another energy-loss edge corresponding to an element present in the specimen is close to the edge of interest. For instance, in our case, the tail of the carbon $\mathrm{K}$ edge at $284 \mathrm{eV}$ was disturbing the acquisition of the first reference image necessary to subtract the background for Ag mapping, and we were forced to adapt the thickness of the carbon substrate and the position of the pre-edge windows consequently. Wrong background estimation can generate some artefacts in the final chemical map, misleading conclusions about the specimen. Fortunately, some techniques have been developed to overcome this problem (Egerton, 1996; Reimer, 1995).

Another concern is the shape of the edge corresponding to a given element, which can be either 'saw tooth-like' (more or less blunted !) for most of the L-shell edges of III $^{\text {th }}$ period elements, or 'white line-type' for elements of the $\mathrm{IV}^{\text {th }}$ period (until copper), according to the vocabulary widely used in the EELS community (Egerton, 1996). 'White line' edges are most favorable for EFTEM imaging because of the high number of electrons that can be selected within a narrow energy slit in the dispersion plane of the spectrometer. This is of main importance since the use of a large slit (to collect more electrons in a wider range of energies) hugely decreases the spatial resolution of the chemical map. The choice of the slit width is always a balance between the spatial resolution and the SNR.

As it was pointed out in this paper, it is possible to overcome the above limitations by minimizing the thickness of the substrate and by determining the optimum energy position and 
energy width of the windows (in the chemical map calculation) during the pre- and post-edge signal acquisition. Practically, the EFTEM technique and the criterion defined in this paper can be used for nanoparticles of any sizes, with shell thicknesses over $1 \mathrm{~nm}$.

Other local techniques using the TEM have been used to characterize core-shell nanoparticles. Li et al. (Li, et al., 2005) give an excellent example of how High Angle Annular Dark Field (HAADF) imaging can be used to obtain chemical information over an area of interest containing core-shell AuAg nanoparticles. A joined advantage for EFTEM and HAADF technique compared to HRTEM is that a low index zone axis orientation is not necessary, as they point out in their conclusion. Garcia-Gutierrez et al. (Garcia-Gutierrez, et al., 2004) also use HAADF to evidence the core-shell structure in AuPt through the contrast produced by the strain field between the Au core and the Pt shell, but in cases where the nanoparticles are oriented along a zone axis.

\section{Conclusion}

In summary, the way a system can be detailed in term of core-shell structure at the nanoscale by the EFTEM technique has been fully demonstrated on the CuAg system, naturally forming nonalloyed nanoparticle configurations. The approach presented in this paper can be very useful to a large community of researchers involved in nanostructured core-shell or alloyed systems characterisation (Amalvy et al., 2005; Horiuchi \& Dohi, 2006). EFTEM and HRTEM techniques are shown to be complementary since HRTEM can provide invaluable structural information at the atomic level whereas EFTEM can give a straight answer about the chemical features of the specimen. Like the EDX technique, the use of the EFTEM technique can be either in a routine and qualitative way or, at the opposite, very fine and quantitative, with new developments frequently published to improve the technique (deconvolution, spectrum imaging, drift corrections...) (Egerton, 2003; Grogger et al., 2005). 


\section{References}

Sao-Joao S., Giorgio S., Penisson J. M. et al (2005) Structure and Deformations of Pd-Ni CoreShell Nanoparticles. J. Phys. Chem. B 109:342-347

Skarman B., Nakayama T., Grandjean D. et al (2002) Morphology and Structure of $\mathrm{CuO}_{\mathrm{x}} / \mathrm{CeO}_{2}$ Nanocomposite Catalysts Produced by Inert Gas Condensation: An HREM, EFTEM, XPS, and High-Energy Diffraction Study. Chem. Mater. 14:3686-3699

Toshima N. (2000) Core/shell-structured bimetallic nanoclusters catalysts for visible-light-induced electron transfer. Pure Appl. Chem. 72:317-325

Toshima N., Harada M., Yamazaki Y. et al (1992) Catalytic activity and structural analysis of polymer-protected $\mathrm{Au}-\mathrm{Pd}$ bimetallic clusters prepared by the simultaneous reduction of $\mathrm{HAuCl}_{4}$ and $\mathrm{PdCl}_{2}$. J. Phys. Chem. 96:9927-9933

Favre L., Stanescu S., Dupuis V. et al (2004) Nanostructured thin films from mixed magnetic CoAg clusters. Appl. Surf. Sci. 226:265-270

Zeng H., Li J., Wang Z. L. et al (2004) Bimagnetic Core/Shell FePt/ $\mathrm{Fe}_{3} \mathrm{O}_{4}$ Nanoparticles. Nano Lett. 4:187-190

Chen X., Lou Y., Samia A. C. et al (2003) Coherency Strain Effects on the Optical Response of Core/Shell Heteronanostructures. Nano Lett. 3:799-803

Reiss P., Bleuse J. \& Pron A. (2002) Highly Luminescent CdSe/ZnSe Core/Shell Nanocrystals of Low Size Dispersion. Nano Lett. 2:781-784

Correa-Duarte M. A., Giersig M. \& Liz-Marzan L. M. (1998) Stabilization of CdS semiconductor nanoparticles against photodegradation by a silica coating procedure. Chem. Phys. Lett. 286:497501 
Kim S., Fisher B., Eisler H. J. et al (2003) Type-II Quantum Dots: CdTe/CdSe(Core/Shell) and CdSe/ZnTe(Core/Shell) Heterostructures. J. Am. Chem. Soc. 125:11466-11467

Basu S. \& Chakravorty D. (2006) Optical properties of nanocomposites with iron core-iron oxide shell structure. J. Non-Cryst. Solids 352:380-385

Gaudry M., Cottancin E., Pellarin M. et al (2003) Size and composition dependence in the optical properties of mixed (transition metal/noble metal) embedded clusters. Phys. Rev. B 67:155409155410

Zhu J., Wang Y., Huang L. et al (2004) Resonance light scattering characters of core-shell structure of Au-Ag nanoparticles. Phys. Lett. A 323:455-459

Kim H., Achermann M., Balet L. P. et al (2005) Synthesis and Characterization of Co/CdSe Core/Shell Nanocomposites: Bifunctional Magnetic-Optical Nanocrystals. J. Am. Chem. Soc. $127: 544-546$

Lu Y., Yin Y., Li Z. Y. et al (2002) Synthesis and Self-Assembly of Au@ $\mathrm{SiO}_{2}$ Core-Shell Colloids. Nano Lett. 2:785-788

Garcia-Gutierrez D., Gutierrez-Wing C., Miki-Yoshida M. et al (2004) HAADF study of Au-Pt core-shell bimetallic nanoparticles. Appl. Phys. A 79:481-487

Li Z. Y., Yuan J., Chen Y. et al (2005) Direct imaging of core-shell structure in silver-gold bimetallic nanoparticles. Appl. Phys. Lett. 87:243103-243103

Hodak J. H., Henglein A. \& Hartland G. V. (2000) Coherent Excitation of Acoustic Breathing Modes in Bimetallic Core-Shell Nanoparticles. J. Phys. Chem. B 104:5053-5055

Sader J. E., Hartland G. V. \& Mulvaney P. (2002) Theory of Acoustic Breathing Modes of CoreShell Nanoparticles. J. Phys. Chem. B 106:1399-1402 
Harada M., Asakura K., Ueki Y. et al (1992) Structure of Polymer-Protected Palladium/Platinum Bimetallic Clusters at the Oxidized

State. Extended X-ray Absorption Fine Structure Analysis. J. Phys. Chem. 96:9730-9738

Huang C. C., Yang Z. \& Chang H. T. (2004) Synthesis of Dumbbell-Shaped Au-Ag Core-Shell Nanorods by Seed-Mediated Growth under Alkaline Conditions. Langmuir 20:6089-6092

Yang J., Lee J. Y., Chen L. X. et al (2005) A Phase-Transfer Identification of Core-Shell Structures in Ag-Pt Nanoparticles. J. Phys. Chem. B 109:5468-5472

Cazayous M., Langlois C., Oikawa T. et al (2006) Cu-Ag core-shell nanoparticles: A direct correlation between micro-Raman and electron microscopy. Phys. Rev. B 73:113402-113404

Vitos L., Ruban A. V., Skriver H. L. et al (1998) The surface energy of metals. Surf. Sci. 411:186202

Campbell C. T. (1997) Ultrathin metal films and particles on oxide surfaces: structural, electronic and chemisorptive properties. Surf. Sci. Rep. 27:1-111

Egerton R. F. (1996) Electron energy-loss spectroscopy in the electron microscope. Plenum Publishing Corp., New York

Reimer L. (1995) Electron Spectroscopic Imaging. Springer, Berlin, Heidelberg

Shindo D. \& Oikawa T. (2002) Analytical Electron Microscopy for Materials Science. SpringerVerlag, Tokyo

Ahn C. C. \& Krivanek O. L. (1983) EELS Atlas: A Reference Guide of Electron Energy Loss Spectra Covering all Stable Elements. Gatan Ed., Palo Alto

Mottet C., Goniakowski J., Baletto F. et al (2004) Phase Transitions 77:101 
Henry C. R. (1998) Surface studies of supported model catalysts. Surface Science Reports 31:231325

Amalvy J. I., Percy M. J., Armes S. P. et al (2005) Characterization of the Nanomorphology of Polymer-Silica Colloidal Nanocomposites Using Electron Spectroscopy Imaging. Langmuir 21:1175-1179

Horiuchi S. \& Dohi H. (2006) Nanoimaging and Spectroscopic Analysis of Rubber/ZnO Interfaces by Energy-Filtering Transmission Electron Microscopy. Langmuir 22:4607-4613

Egerton R. F. (2003) New techniques in electron energy-loss spectroscopy and energy-filtered imaging. Micron 34:127-139

Grogger W., Varela M., Ristau R. et al (2005) Energy-filtering transmission electron microscopy on the nanometer length scale. J. Electron Spectrosc. Relat. Phenom. 143:141-149 


\section{Figure Captions}

Figure 1. (a) $\mathrm{Cu} \mathrm{L}_{2,3}$ edge, and (b) $\mathrm{Ag} \mathrm{M}_{4,5}$ edge. Spectra are obtained by selecting a large area on the B specimen. Background has been subtracted on the two spectra.

Figure 2. (a) Ag chemical map of an area on sample A, (b) Cu chemical map of the same area and (c) Color-coded chemical map with $\mathrm{Ag}$ (blue) and $\mathrm{Cu}$ (yellow). Note the presence of pure $\mathrm{Ag}$ nanoparticles that have nucleated separately on the substrate.

Figure 3. (a) EFTEM image of sample A, together with intensity profiles corresponding respectively to a core-shell particle (left) and a pure Ag particle (right). Profile positions are reported on the EFTEM image (white rectangles). (b) Geometrical transverse profile of a supported core-shell nanoparticle.

Figure 4. Statistical measurements on sample A - a) Size distribution showing the superimposed distribution of pure $\mathrm{Ag}$ nanoparticles and $\mathrm{CuAg}$ nanoparticles, and b) shell thickness distribution (spatial error for the shell thickness measurements: $1 \mathrm{~nm}$ approximately).

Figure 5. High resolution image of a core-shell nanoparticle of sample A, oriented along the [111] systematic row, showing large and dark moiré fringes superimposed. The inset of the figure shows the power spectrum of the image.

Figure 6. (a) Ag chemical map obtained by EFTEM technique on sample B, (b) size distribution showing the superimposed distribution of pure $\mathrm{Ag}$ nanoparticles and $\mathrm{CuAg}$ nanoparticles, and (c) shell thickness distribution (spatial error for the shell thickness measurements: $1 \mathrm{~nm}$ approximately).

Figure 7. (a) HRTEM image, (b) Bragg filtered image and (c) EFTEM image of the same nanoparticle oriented along the [110] zone axis. 